\title{
Onboard Atomic Clock Frequency Offset for Indian Regional Navigation Satellite System
}

\author{
Babu R., Rethika T., and Rathnakara S. C.
}

\begin{abstract}
The IRNSS (Indian Regional Navigation Satellite System) user receiver calculates its position using the timing information embedded in the navigation signal, transmitted from the IRNSS satellites. The timing information broadcasted in the navigation signal is derived from Atomic clock onboard the IRNSS satellite. A perfect and high stability frequency source is required for precise time measurement. Highly accurate time synchronization between satellite time and ground system time is also required which is synchronized through uplink broadcast navigation parameters. But there is a shift in the frequency of the atomic clock when it is transported from ground to the satellite orbit. Since the atomic clock frequency onboard the IRNSS spacecraft has a very profound impact on user position accuracy, it is appropriate to know what should be the exact frequency of atomic clock to be set before satellite launch. This frequency has to be computed by taking into account the various relativistic effects on the satellite clocks. In this paper, we compute this offset (Factory offset) for the IRNSS satellite clocks that will be set before launch of the IRNSS satellites.
\end{abstract}

Index Terms-IRNSS, relativistic effects, satellite clocks

\section{INTRODUCTION}

Indian Regional Navigation Satellite System (IRNSS) envisages establishment of regional navigational satellite system using a combination of GEO (Geo Stationary Orbit) and GSO (Geo Synchronous Orbit) Satellites [1]. IRNSS is expected to provide position accuracy better than 20 meters over India and a region extending about $1500 \mathrm{~km}$ around Indian geo-political boundary.

The IRNSS time (IRNWT) is determined from a clock ensemble composed of the cesium and hydrogen maser atomic clocks at the Indian Navigation Centre (INC) ground stations. As with UTC, IRNWT is also a weighted mean average time but with two substantial differences. IRNWT will be made available in real time and is continuous time without leap seconds. The IRNSS satellites carries rubidium atomic frequency standard onboard. At INC through navigation software these onboard clocks are monitored and controlled. The deviation between each of the satellite and IRNWT is modeled with a quadratic function of time, and the parameters of this model are calculated and transmitted as a part of the IRNSS broadcast navigation messages. The parameters are often called as clock bias (A0) or the clock offset (in seconds), drift (A1) or the relative frequency instability (in seconds/second) and aging (A2) also referred

Manuscript received April 13, 2012; revised May 25, 2012.

The authors are with the Space Navigation Group, ISRO Satellite Center, Bangalore-560 017, India (e-mail: rbabu@isac.gov.in, rethika@isac.gov.in, ratnakar@isac.gov.in) as relative frequency shift (in seconds/seconds ${ }^{2}$ ). Apart from these corrections any IRNSS users should considers the necessary relativistic time adjustment. With these adjustment parameters, which are usually calculated once per day is then transmitted to the satellites, thus the satellite clock errors are expected to be well within 5-10ns which fulfills the requirement.

According to Einstein's theory of relativity, the satellite orbiting the earth will experience two major relativistic influences upon its rate of timekeeping: a special relativistic correction for its orbital speed and a general relativistic correction for its orbital altitude. First, the transmitting clock is subject to time dilation due to its orbital speed. Second, a clock at the higher gravitational potential of orbit runs faster than a surface clock. Hence a clock in circular orbit with a radius of $36000 \mathrm{Km}$ would gain few micro seconds per day compared to clocks on Earth's surface. In order to compensate for this, the IRNSS onboard clocks are not tuned to the nominal frequency of $10.23 \mathrm{MHz}$, but are set to a different frequency which is reduced by the so called "factory offset".

Although clock velocities are small and gravitational fields are weak, they give rise to significant relativistic effects. These effects include first-order and second-order Doppler frequency shifts of clocks due to their relative motion, gravitational frequency shifts, and the sagnac effect due to earth's rotation. These effects are to be accounted for to achieve precise navigation and time transfer solutions.

The major objective of this work is to bring out how relativistic effects accounted for the IRNSS and hence compute the factory frequency offset for IRNSS satellite clocks.

\section{FREQUENCY OFFSET FOR SATELlite ClOCKS}

\section{A. Fundamental Frequency}

The inertial coordinate system at rest, its origin located at the center of the earth is taken as reference to view all IRNSS related activities. Because of the motion velocities and slightly eccentric orbits of the IRNSS satellites, the nonnegligible gravitational potential difference between the satellite and the users, as well as the rotation of the earth, the relativistic effects have to be taken into account.

The fundamental frequency $f_{0}$ of the IRNSS system is selected as 10.23 MHz [2]. All clocks on the IRNSS satellites and IRNSS receivers operate based on this frequency. If all the IRNSS satellites are working simply on the frequency $f^{\prime}=f_{0}$, then we will view a frequency $f$ at our reference point, and $f$ is not the same as $f_{0}$ due to relativistic effects. In order to able to view the fundamental 
frequency $f=f_{0}$ the desired working frequency $f^{\prime}$ of the IRNSS satellite clock can be computed. The two major offsets affecting a satellite clock has to be accounted for, in achieving the desired working frequency of the IRNSS system.

\section{B. Major Offsets for a Satellite Clock}

The two major relativistic effects on IRNSS satellite clocks are due to

- Gravitational potential difference at high altitude. As a result of which, the satellite clocks run faster when compared to clocks on ground.

- Orbital motion of satellites. As a result of which, the satellite clocks run slower when compared to clocks at rest on ground.

1) Offset due to gravitational potential difference

Gravitational frequency shifts of clocks on board IRNSS satellites arises due to the fact that a reference clock at rest on the earth's surface at the equator and an atomic clock on board a satellite in space do not experience the same gravitational potential. A clock in a weaker gravitational potential appears to run fast in comparison to one that in a stronger gravitational potential [3].

Offset due to gravitational potential is given by $-\frac{\phi_{0}}{c^{2}}$

where $\phi_{0}$ is the reference potential and it is given by,

$$
\phi_{0}=-\frac{\mathrm{GM}_{\mathrm{e}}}{\mathrm{R}_{\mathrm{e}}}\left(1+\frac{\mathrm{J}_{2}}{2}\right)-\frac{1}{2} \Omega_{\mathrm{e}}^{2} \mathrm{R}_{\mathrm{e}}^{2}
$$

where, $G M_{e}$ is Earth gravitational constant,

$R_{e}$ is Mean earth radius at equator,

$J_{2}$ is Earth's quadrupole moment,

$\Omega_{e}^{2}$ is Earth angular rotation rate.

(Note: For the values of these constants refer appendix)

\section{2) Offset due to Orbital velocity}

There is a frequency shift in the clock on board the IRNSS satellites due to their motion through an EarthCentered Inertial (ECI) frame. This effect arises due to the phenomenon of time dilation which is a consequence of the special theory of relativity. This effect actually slows down the satellite clocks [3].

Offset due to velocity of satellite is given by $-\frac{3 \mathrm{GM}_{\mathrm{e}}}{2 \mathrm{ac}^{2}}$

where, $G M_{e}$ is Earth gravitational constant.

a is Semi-major axis of satellite orbit.

c is velocity of light.

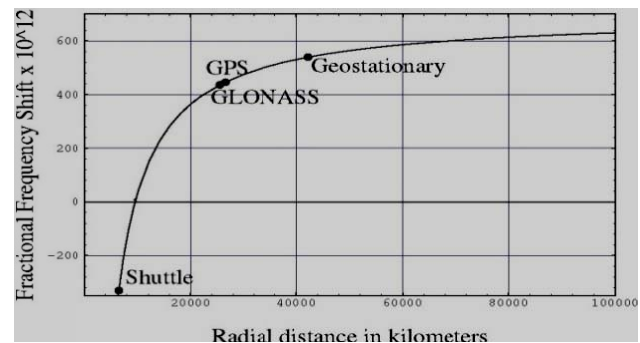

Fig. 1. Net fractional frequency shift of a clock in various orbits.

\section{Frequency Shift for Satellites in Various Orbits}

The following plot shows the fractional frequency shift for satellites having different radial distances [4]:

We now proceed with the computation of factory offset for IRNSS in the next section (section: 3 )

\section{COMPUTATION OF FACTORY OFFSET FOR IRNSS}

The factory frequency offset for IRNSS is computed by taking into account both the relativistic effects due to gravitational potential difference and orbital motion of satellite clocks as shown below:

\section{A. Offset Due to Gravitational Potential Difference}

The offset due to earth's gravitational potential for IRNSS sis calculated as shown below. The equation for reference potential (from 2) is,

Reference potential, $\phi_{0}=-\frac{G M_{e}}{R_{e}}\left(1+\frac{J_{2}}{2}\right)-\frac{1}{2} \Omega_{e}^{2} R_{e}^{2}$

where $G M_{e}$ is Earth gravitational constant,

$R_{e}$ is Mean earth radius at equator,

$J_{2}$ is Earth's quadrupole moment,

Substituting the values for the constants from the appendix,

$$
\text { We get, } \begin{array}{r}
\phi_{0} \\
\frac{\phi_{0}}{\mathrm{c}^{2}}=-6.2636803 \times 10^{7} \mathrm{~m}^{2} / \mathrm{s}^{2} \\
-\frac{\phi_{0}}{\mathrm{c}^{2}}=+6.9692842 \times 10^{-10}
\end{array}
$$

This is the required fractional frequency offset for IRNSS satellite clocks due to gravitational potential difference because of the transport of clock from ground to orbital altitude.

\section{B. Offset Due to Velocity of IRNSS Satellites}

The offset due to velocity of satellite is calculated as shown below,

Offset due to velocity of satellite (from 3 ) is given by $-\frac{3 \mathrm{GM}}{2 \mathrm{ac}^{2}}$

Substituting the values of constants from the appendix, we get,

$$
-\frac{3 G M}{2 a c^{2}}=-1.577777738 \times 10^{-10}
$$

This is the required fractional offset for IRNSS satellite clocks due to orbital velocity of the clock while in orbit.

\section{Factory Offset Computation}

The net fractional frequency offset due to the above two effects is computed as below:

Fractional clock rate offset is given by, $-\frac{3 \mathrm{GM}_{\mathrm{e}}}{2 \mathrm{ac}^{2}}-\frac{\phi_{0}}{\mathrm{c}^{2}}=$ $-1.577777738 \times 10^{-10}+6.9692842 \times 10^{-10}$

$$
=+539.1506499 \times 10^{-12}
$$

where, $\quad \alpha=$ semi major axis (taken as $42164 \mathrm{~km}$ )

$$
\mathrm{GM}_{\mathrm{e}}=3.986004415 \times 10^{14} \mathrm{~m}^{3} / \mathrm{s}^{2}(\mathrm{JGM}-2)
$$

The fractional value obtained $539.1506499 \times 10^{-12}$ coincides with that for geostationary satellites as observed from the net fractional frequency shift figure (Fig.1).

Frequency to be offset is given by, 


$$
\begin{array}{r}
5.391506499 \times 10^{-10} \times 10.23 \times 10^{6} \\
=0.005515511148 \mathrm{~Hz}
\end{array}
$$

Relativistic effect is compensated by setting IRNSS clocks at frequency

$$
\begin{gathered}
=10.23 \times 10^{6}-0.005515511148 \mathrm{~Hz} \\
=10.229999994484488852 \mathrm{MHz}
\end{gathered}
$$

\section{Correction Term for Eccentric Orbit}

IRNSS satellites have a slightly eccentric orbit. Hence the relativistic effects due to eccentric orbits have to be taken care in IRNSS. The additional correction term for eccentric orbits is made at the receiver end by adding the correction term given by,

$$
\Delta t_{r}=F e \sqrt{a} \sin E_{k}
$$

where $\mathrm{F}=-\frac{2 \sqrt{\mu}}{\mathrm{c}^{2}}=-4.442807633 \times 10^{-10} \frac{\mathrm{sec}}{\sqrt{\text { meter }}}$

$$
\begin{aligned}
& e=\text { Eccentricity } \\
& \mathrm{a}=\text { semi major axis } \\
& E_{k}=\text { Orbital parameter in Kepler's equation of }
\end{aligned}
$$
eccentric anomaly

Hence,

$$
\Delta \mathrm{t}_{\mathrm{r}}=+4.442807633 \times 10^{-10} \text { e } \sqrt{\mathrm{a}} \sin \mathrm{E}_{\mathrm{k}}
$$

\section{IV.CONCLUSION}

Relativistic effects should be considered for IRNSS satellites while precise time and frequency measurements are involved. Hence a factory offset for IRNSS atomic clocks has been computed taking into account of the relativistic effects affecting them while in motion in an orbit at a high altitude. Based on the calculations made, atomic clock frequency for IRNSS satellites should be set to 10.229999994484488852 MHz before launch of satellite.

\section{APPENDIX}

\section{A. Constants Used in the Computation}

TABLE I: TABLE OF CONSTANTS

\begin{tabular}{|c|c|c|c|}
\hline $\begin{array}{c}\text { S. } \\
\text { No }\end{array}$ & Parameter & value & units \\
\hline 1 & Speed of light $(\mathrm{c})$ & $2.99792458 \times 10^{8}$ & $\mathrm{~m} / \mathrm{s}$ \\
\hline 2 & $\begin{array}{c}\text { Universal gravitational } \\
\text { constant }(\mathrm{G})\end{array}$ & $6.6732 \times 10^{-11}$ & $\begin{array}{c}\mathrm{m}^{3} / \mathrm{kg} \\
\mathrm{s}^{2}\end{array}$ \\
\hline 3 & $\begin{array}{c}\text { Earth's quadrupole } \\
\text { moment }\left(\mathrm{J}_{2}\right)\end{array}$ & $1082.6269 \times 10^{-6}$ & - \\
\hline 4 & Mass of earth $\left(\mathrm{M}_{\mathrm{e}}\right)$ & $5.9742 \times 10^{24}$ & $\mathrm{~kg}$ \\
\hline 5 & $\begin{array}{c}\text { Mean earth radius at } \\
\text { equator }\left(\mathrm{R}_{\mathrm{e}}\right)\end{array}$ & $6.3781363 \times 10^{6}$ & $\mathrm{~m}$ \\
\hline 6 & $\begin{array}{c}\text { Earth gravitational constant } \\
\left(\mu=\mathrm{GM}_{\mathrm{e}}\right)\end{array}$ & $3.986004415 \times 10^{14}$ & $\mathrm{~m} / \mathrm{s}^{2}$ \\
\hline 7 & $\begin{array}{c}\text { Earth angular rotation rate } \\
\left(\Omega_{\mathrm{e}}\right)\end{array}$ & $\begin{array}{c}7.2921151467 \\
\times 10^{-5}\end{array}$ & $\mathrm{rad} / \mathrm{s}$ \\
\hline
\end{tabular}

\section{REFERENCES}

[1] Global Indian Navigation Satellites: Constellation studies, August ISRO-ISAC-IRNSS-TR-0887, 2009.

[2] Indian Regional Navigation Satellite system signal in space ICD, Nov 2009, ISRO-ISAC-IRNSS-SPS-RS/1.0, 2009.

[3] Global positioning system: Theory and applications. Bradford W. Parkinson, James J. Spilker Jr. (Ch: 18)

[4] Relativity and the Global Positioning System, Neil Ashby 